\title{
DNAssist, a C++ Program for Editing and Analysis of Nucleic Acid and Protein Sequences on PC-Compatible Computers Running Windows 95, 98, NT4.0 or 2000
}

BioTechniques 28:1192-1197 (June 2000)

\author{
Hugh G. Patterton and Steven Graves ${ }^{1}$ \\ University of Cape Town, Rondebosch, South \\ Africa, and ${ }^{1}$ Los Alamos National Laboratories, \\ Los Alamos, NM, USA
}

\begin{abstract}
The size of sequence databases, the rapid rate of generating sequence data and the need to routinely construct recombinant DNA molecules in molecular biological research necessitate the frequent handling and analysis of nucleotide and protein sequences on a computer. Although several console or Web-based utility programs are available, the application of these programs generally requires reformatting the data when proceeding from one such program to the next. The acquisition of elaborate, integrated program suites is financially prohibitive to smaller laboratories. Here, we report the development of DNAssist, a shareware program for editing and analysis of nucleic acid and protein sequences. It was developed as a multiple-document interface program-similar to a word processor-where sequences are entered, edited and analyzed in a single integrated environment. DNAssist can calculate the physicochemical properties of a sequence, convert between nucleic acid and protein sequences, translate DNA in multiple frames, identify open reading frames and locate ambiguous sequence patterns allowing gaps and mismatches. DNAssist also performs restriction enzyme and transcription factor-binding site analyses of DNA sequences, the multiple alignment of nucleic acid and protein sequences and the analysis of DNA sequences for nucleosome positioning sites.
\end{abstract}

\section{INTRODUCTION}

Research in molecular biology generally requires microcomputers to edit, manipulate and analyze nucleotide and protein sequences. The availability of the sequence of the entire genome of some organisms $(3,20)$, the rapid generation of sequence data and the requirement to design and construct re- combinant DNA molecules in research have made it necessary for almost all molecular biological laboratories to store and analyze sequences on computers. Common analyses include the identification of specific and functionally significant subsequences, the conversion of one sequence type to another, the location of consensus sites such as those for restriction enzymes or for transcription factors and the multiple alignment of sequences. Often the physicochemical properties such as molecular weight, melting temperature, isoelectric point, molar absorption coefficient at a given wavelength and the composition of polynucleotides and polypeptides are also required.

These analyses can be performed with individual utility programs that are often console-based (19), form-based Web interfaces to server-side Perl or C scripts (22) or with larger program suites such as GCG (7) that require mainframe or workstation computers running UNIX ${ }^{\circledR}$ or Solaris ${ }^{\circledR}$ operating systems. However, when performing a series of analyses with separate utility programs, the data are generally required or generated in specific formats and need to be reformatted in a text-based editor when proceeding from one analysis program to the next. The acquisition of sophisticated program suites that offer an integrated environment for analysis is often financially prohibitive to many smaller research laboratories. Here, we report the development of DNAssist, a shareware C++ program designed with a multiple-document interface (MDI) with integrated editing and extensive analysis capabilities that can be used on a PC-compatible computer running the Microsoft ${ }^{\circledR}$ Windows ${ }^{\circledR} 95,98$, NT $^{\circledR}$ version 4.0 or 2000 operating system.

\section{MATERIALS AND METHODS}

\section{Program Design}

DNAssist is coded in $\mathrm{C}++(1,18)$ and the graphic user interface developed using version 5.02 of the Borland ${ }^{\circledR}$ Objects Windows Library (Inprise, San Jose, CA, USA). The code was compiled with version 5.02 of the Borland ${ }^{\circledR} \mathrm{C}++$ compiler (Inprise) for Microsoft Windows 95, 98, NT4.0 or Windows 
2000, and was optimized for execution speed; C++ exceptions were enabled. The code is object orientated, with classes designed for sequence storage, management, manipulation, analysis and presentation (a paper detailing the design and relationship between classes will be published elsewhere). The proper allocation and de-allocation of memory blocks by the program was tested with version 2.0 of Borland ${ }^{\circledR}$ Codeguard $^{\circledR}$ (Inprise), and the program was subjected to three beta testing phases in unrelated molecular biology laboratories. DNAssist requires an Intel Pentium MMX or related CPU with $32 \mathrm{MB}$ RAM and at least $15 \mathrm{MB}$ available disk space for installation; it can be downloaded from http://www.dnassist.com.

\section{RESULTS AND DISCUSSION}

\section{General Program Layout}

DNAssist is designed as an MDI, as shown in Figure 1. The user is presented with a familiar environment, similar to that of a word processing program, in which sequences are managed and edited and analyses performed. All file and windows management, sequence editing and analysis operations may be selected from the menu, where relevant methods and commands are logically grouped under the File, Edit, Convert, Analyze, Options, Windows and Help menu items. The more frequently used commands are also accessible from buttons on a movable toolbar (Figure 1) that displays hints when the mouse pointer is moved over individual buttons. All the analysis methods can be selected from the Analysis menu or from a context-sensitive pop-up menu. When a method is selected, it is directly applied to a displayed sequence or sequence selection in the editor window with input focus. The user can analyze multiple types of sequences in a myriad of ways within one easy-to-use integrated environment.

The user can open several windows simultaneously in which sequences (editor windows) or the results of analyses (result windows) are displayed. The windows can be resized and arranged using the mouse pointer or the Windows menu, which also lists all open editor and result windows (Figure 1).

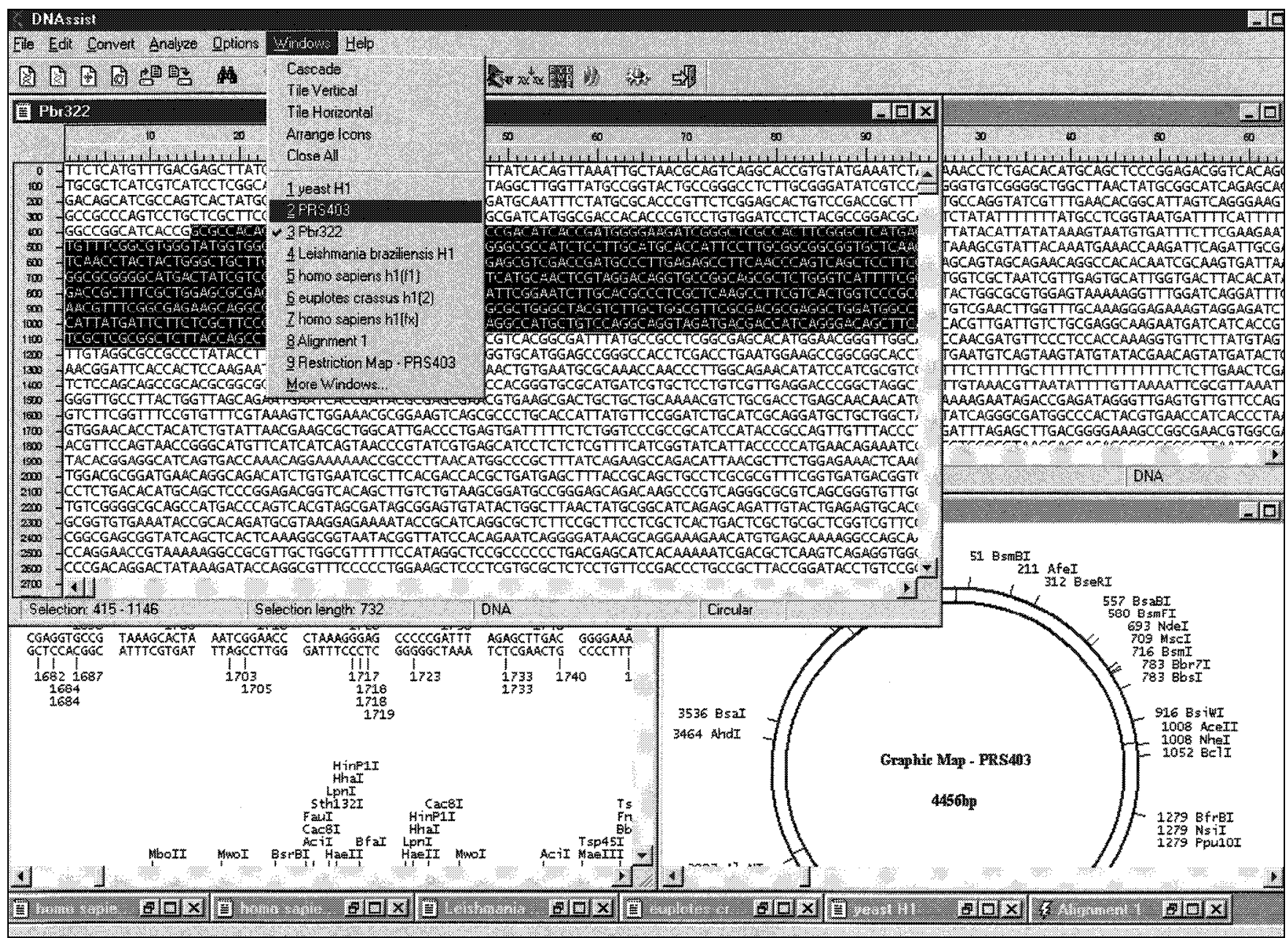

Figure 1. The DNAssist sequence editing and analysis environment. DNAssist is designed as an MDI program that allows the user to have multiple-sequence editing and analysis-result windows open simultaneously. These windows can be resized, arranged or minimized. All analysis methods can be selected from the menu and are directly applied to the sequence or sequence selection in the editor window with input focus. Frequently used actions are also accessible from the toolbar and from a context sensitive pop-up menu that is displayed by clicking the right mouse button. 


\section{Sequence Editor}

There are four different editor-type windows in which to display and edit DNA or degenerate DNA, RNA or protein sequences. New editor windows can be opened from the File menu or from the toolbar. The sequence editor window depends on the type chosen by the user from the menu, in the case of a new editor window, or on the sequence type of a file opened from a disk. For sequence files that do not specify a sequence type, such as text or FASTA (15) format files, DNAssist assigns a type that recognizes the largest percentage of characters in the sequence file.

The user can enter a sequence from the keyboard or load a sequence from a disk if the file is in text, FASTA (15), GenBank $^{\circledR}$ (16), GCG (7), DNA Strider (13) or DNAssist format. The editor window responds to all uppercase or lowercase character keys that represent legitimate symbols for the window sequence type, general editing keys such as Delete and Backspace and the navigation arrow buttons. In addition, several shortcut keystrokes for common editing actions such as Copy, Cut and Paste are also defined. Alternatively, sequences can be dragged and dropped within (or between) compatible editor window types or pasted from the clipboard into a compatible window. Selections in a sequence can be made using the keyboard or the left button on the mouse and dragging the pointer within an editor window. The user can specify the font face and size of installed fixed pitch fonts, as well as the line length displayed in an editor window from the Options menu. All editor and analyses settings specified by the user are saved in a unique subkey of HKEY_CURRENT_USER in the Registry when the user exits the program, a feature that allows DNAssist to be used in a multi-user environment.

A sequence displayed in an editor window is manipulated in memory as a continuous series of eight-bit character data types and is managed by a specialized $\mathrm{C}++$ class. This class will dynamically expand or shrink the allocated memory block in which the character array is placed in response to additions or deletions to the sequence. This allows optimum memory use and the manipulation of sequences with a size constrained only by the physical configuration of the computer.

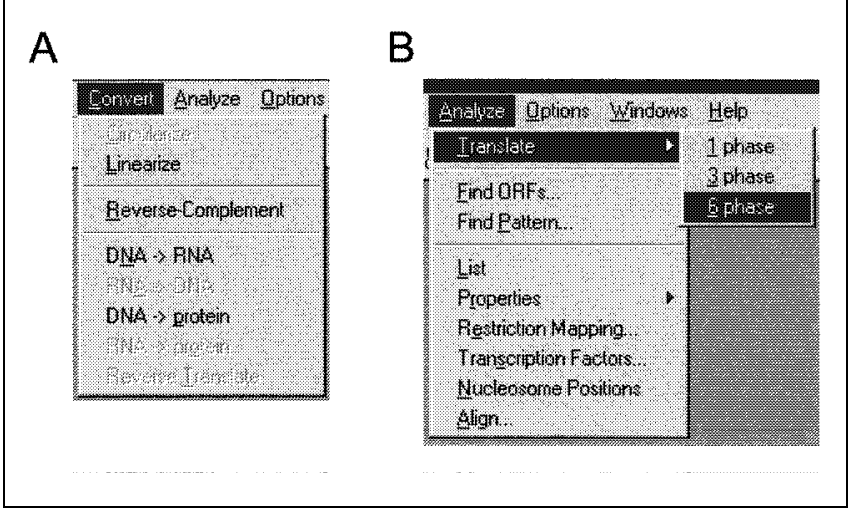

Figure 2. Sequence conversions and translations in DNAssist. (A) Nucleic acid sequences can be reversibly interconverted or converted to a protein sequence from the Convert menu. (B) DNA sequences can be translated in 1, 3 or all 6 reading frames from the Analyze menu.
Sequences can be saved to disk in ASCII text or DNAssist format. The format file of the latter contains a file header of five semicolon-delimited fields, in which information regarding the stored sequence is given and is followed by a series of bytes that correspond to the ASCII character values of the nucleotides or amino acid residues in the sequence.

\section{Sequence Analysis}

The analysis methods that can be selected from the main menu or the context-sensitive pop-up menu depend on the sequence type of editor window with input focus. The menu entries and toolbar icons of analysis methods that cannot be applied to a given sequence type are dimmed to present the user with a more intuitive graphic interface.

The result of an analysis is displayed in a result MDI window. In the case of a text-based result, the content of the result window can be saved to a disk or copied to the clipboard in rich-text format (RTF), from which it can be opened or pasted into other applications that recognize this format. Similarly, graphic results can be saved or copied and imported into other applications that recognize the enhanced Windows metafile format. The user has extensive control over the format in which text-based results are displayed. In the case of sequence listings, one or both DNA strands can be shown as a continuous

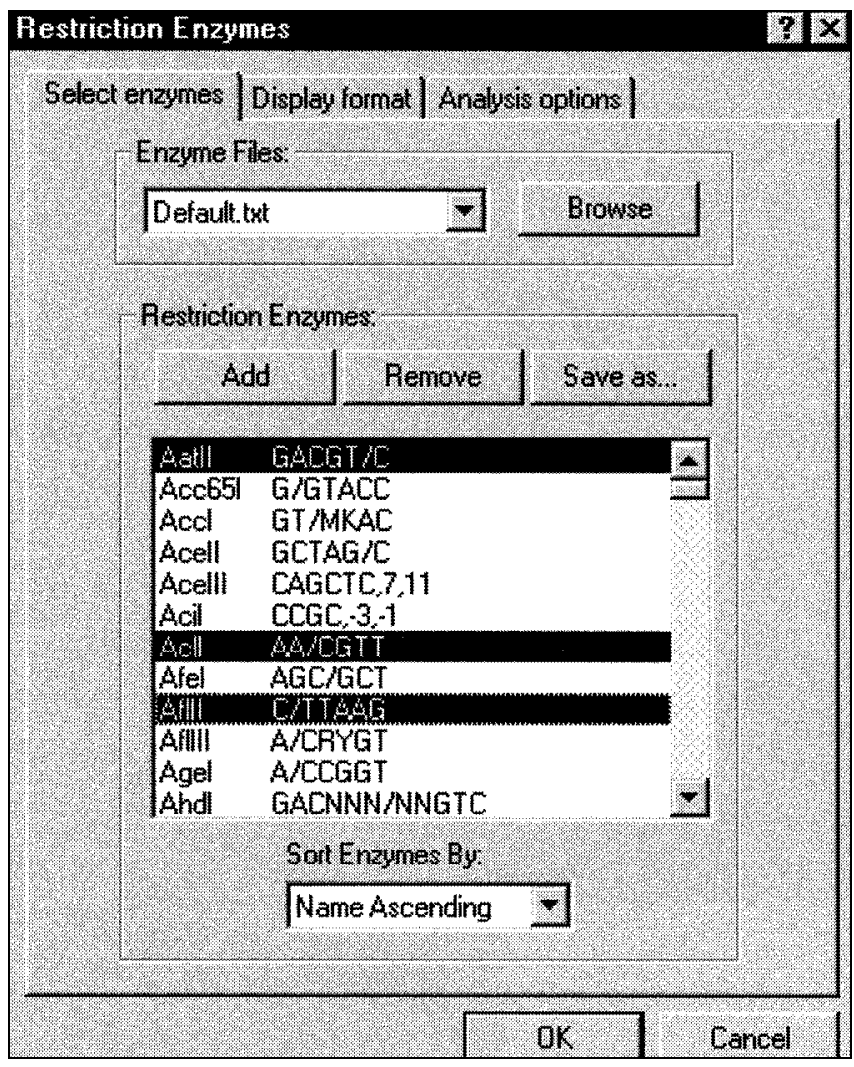

Figure 3. Restriction enzyme analysis. All restriction enzymes in a specified file are listed in the Restriction Enzymes dialog box. Individual restriction enzymes can be deleted, new enzymes added and the modified file saved to disk. The user can also select specific restriction enzymes from the enzyme list. 


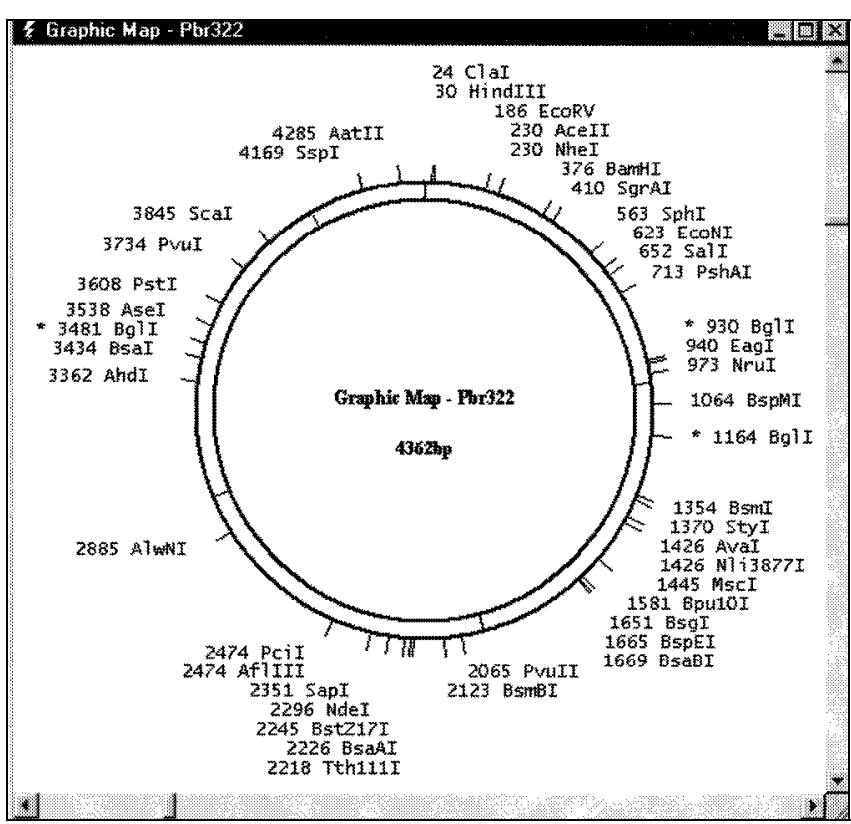

Figure 4. The display of a restriction enzyme analysis. A graphic map of a restriction enzyme analysis will display all unique sites and, if specified by the user, will indicate the presence of a cleavage site for a selected restriction enzyme, with an asterisk. sequence or grouped into sequence blocks with a block size and block spacing that may be specified. Sequence numbering can be displayed above, below or to the right of such listings.

\section{Sequence Properties}

DNAssist can calculate and display the general physicochemical properties of nucleic acid and protein sequences, plus the hydrophobicity (12), hydrophilicity (11) and antigenicity (21) profiles of protein sequences. The following are displayed for nucleic acid sequences: the number of each of the four nucleotides, $\mathrm{G}+\mathrm{C} \%$, A+T\%, sequence length, melting temperature calculated by the nearest neighbor method (6), annealing temperature, molecular weight, molar absorption coefficient and $\mathrm{E}_{0.1 \%}$ at $260 \mathrm{~nm}$ (2). Displayed for protein sequences are the number and mole percentage of each of the 20 amino acid residues, sequence length, molecular weight, pI (4), molar absorption coefficient and $\mathrm{E}_{0.1 \%}$ at 280 $\mathrm{nm}$ (14). These properties can be displayed for the entire sequence or for only a selected subset of the sequence.

\section{Sequence Reversals, Conversions and Translations}

A DNA sequence can be transformed between a linear and circular configuration or converted to its complementary 
sequence in the $5^{\prime}$ to $3^{\prime}$ orientation. This conversion takes place as a combined reverse-complement operation. A nucleic acid sequence can also be converted to a protein sequence, DNA and RNA sequences can be interconverted (Figure 2A) and a DNA sequence can be translated to a protein sequence in one, three or all six frames (Figure 2B). In addition, a protein sequence can also be reverse translated to a degenerate DNA sequence. Nucleic acid to protein conversions and translations are performed according to the entries in the codon table selected from the Options menu.

Codon tables generated by the GCG program, Codonfrequencies (7), are compatible with DNAssist. A translated sequence is displayed as a nucleotide sequence listing aligned with the single-letter amino acid abbreviation. Stop codons are indicated by asterisks. If an in-frame stop codon is encountered during a nucleic acid-to-protein conversion, the user is alerted to the truncation of the protein at the given position.

\section{Finding ORFs and Patterns}

All the sequence searches in DNAssist make use of a class implementation of the Boyer-Moore algorithm (5). Apart from routine substring searches from the Edit menu, DNAssist also allows searching of a sequence for ORFs and se- quence patterns. The ORF search function uses the start and stop codons in the selected codon table to search for continuous sequence stretches that may code for proteins. The pattern-search function searches for a defined sequence pattern, allowing ambiguous nucleotides or amino acid residues in the pattern and a user-defined number of sequence gaps and mismatches. The sequence of an identified ORF or pattern match is highlighted in the editor window.

\section{Restriction Enzyme Analysis}

DNAssist performs a restriction enzyme analysis using restriction enzyme data from a Rebase database file (17). Updated versions of this enzyme data file can be downloaded from the Rebase Web site at http://rebase.neb.com/rebase/ rebase.files.html. All or a selection of the restriction enzymes in the file can be used (Figure 3), and enzymes leaving a specific type of cleaved terminus can also be specified from this set. The result of a restriction enzyme analysis can be displayed as a graphic map (Figure 4), a sequence listing and in tabulated format. The tabulated restriction enzyme analysis lists the enzymes used in the analysis, the number of times that each enzyme cleaves, the cleavage positions and fragment sizes in descending order and a list of restriction enzymes that do not have recognition sequences in the analyzed sequence.

\section{Predicting Nucleosome Rotational Positions}

The propensity of a DNA sequence to accommodate a rotationally positioned nucleosome is calculated with the DrewCalladine algorithm (8) and is displayed as a plot of the probability of finding a rotationally positioned nucleosome at every position of the analyzed sequence.

\section{Sequence Alignments}

DNAssist conducts pairwise and multiple alignments of DNA, degenerate DNA, RNA and protein sequences by constructing a phylogenetic tree that is based on a matrix of all pairwise sequence similarity scores, as used in CLUSTALW $(10,19)$. The alignment allows ambiguous nucleotides or

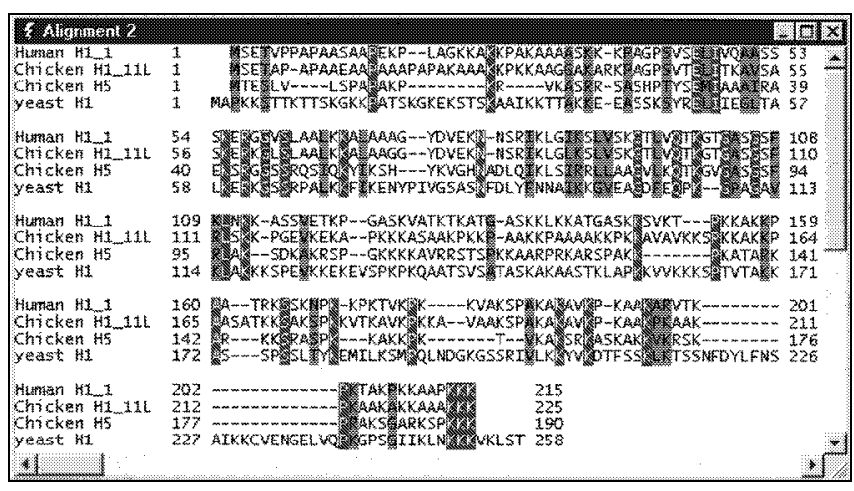

Figure 5. Multiple alignments in DNAssist. A multiple alignment is displayed with conserved and identical amino acid residues or identical nucleotides, as shown in a selected font foreground and font background color. The minimum number of nucleotides or amino acid residues that must be identical or conserved to be indicated as such can also be specified. 
amino acid residues. Aligned sequences are displayed as sequence lists with identical nucleotides and amino acid residues or as conserved amino acid residues, indicated in specified font foreground and background colors (Figure 5). From the Options menu, the user can specify the colors and the minimum level of sequence identity or conservation required before residues are indicated as such. The user can also specify Gap extension, opening penalties and the minimum level of divergence before alignment is delayed. DNA sequence alignments can be performed with a user-selected DNA weight matrix, and the penalty associated with a nonidentical purine-purine or pyrimidine-pyrimidine alignment can be set. For protein sequences, the protein weight matrix and penalties for opening gaps at specific residues, sequence termini or hydrophilic regions are also user selectable.

\section{Promoter Analysis for Transcription Factor Binding Sites}

A DNA sequence can be analyzed for the presence of transcription factor binding sites listed in the Transfac database (9). DNAssist lists transcription factors in the database in a hierarchic manner, grouped by kingdom, species and factor. These groups are displayed in list boxes with a master-slave relationship. For example, the user can select all vertebrate factors or only the factors identified in Homo sapiens. DNAssist issues a local structured query language (SQL) query against the Transfac database and displays only the factors returned for the selected kingdom(s) and species. The user can then choose to perform the analysis with all or only a selection of displayed transcription factors. Updated versions of the Transfac database can be downloaded from http://transfac.gbf.de/TRANSFAC/index.html. The result of a transcription factor analysis can be displayed as a graphic map, sequence listing or in tabulated form, where the name and binding position of the selected transcription factors are given.

\section{SUMMARY}

DNAssist is an excellent tool set for the molecular biologist while remaining affordable for the research scientist. As such, it is a marked advance over other such tools on the market that cost as much as 10 times more. In today's atmosphere of increasingly tight funding, DNAssist is an essential and cost-effective program for almost all molecular biology researchers.

\section{ACKNOWLEDGMENTS}

The authors thank Andrew Flaus and Allison Johnson for their many helpful suggestions.

\section{REFERENCES}

1.American National Standards Institute. 1998. Information TechnologyProgramming Languages-C++. Document number ISO/IEC 14882-1998. American National Standards Institute, Bethesda, MD.

2.Ausubel, F., R. Brent, R.E. Kingston, D.D. Moore, J.G. Seidman, J.A.
Smith and K. Struhl. 1995. Short Protocols in Molecular Biology. Third Edition, John Wiley and Sons, New York.

3.Blattner, F.R., G. Plunkett, C.A. Bloch, N.T. Perna, V. Burland, M. Riley, J. Collado-Vides, J.D. Glasner et al. The complete genome sequence of Escherichia coli K-12. Science 277:1453-1474.

4.Bjellqvist, B., B. Basse, E. Olsen and J.E. Celis. 1994. Reference points for comparisons of two-dimensional maps of proteins from different human cell types defined in a $\mathrm{pH}$ scale where isoelectric points correlate with polypeptide compositions. Electrophoresis 15:529-539.

5.Boyer, R.S. and J.S. Moore. 1977. A fast string searching algorithm. Commun. ACM. 20:762-772.

6.Breslauer, K.J., R. Frank, H. Blocker and L.A. Marky. 1986. Predicting DNA duplex stability from the base sequence. Proc. Natl. Acad. Sci. USA 83:3746-3750.

7.Devereux, J., P. Haeberli and O. Smithies. 1984. A Comprehensive Set of Sequence Analysis Programs for the VAX. Nucleic Acids Res. 12:387395.

8.Drew, H.R. and C.R. Calladine. 1987. Sequence-specific positioning of core histones on an 860-base-pair DNA. Experiment and theory. J. Mol. Biol. 195:143-173.

9.Heinemeyer, T., X. Chen, H. Karas, A.E. Kel, O.V. Kel, I. Liebich, T. Meinhardt and I. Reuter. 1999. Expanding the TRANSFAC database towards an expert system of regulatory molecular mechanisms. Nucleic Acids Res. 27:318-322.

10.Higgins, D.G. and P.M. Sharp. 1988. CLUSTAL: a package for performing multiple sequence alignment on a microcomputer. Gene 73:237244.

11.Hopp, T.P. and K.R. Woods. 1981. Prediction of protein antigenic determinants from amino acid sequences. Proc. Natl. Acad. Sci. USA 78:3824-3828.

12.Kyte, J. and R.F. Doolittle. 1982. A simple method for displaying the hydropathic character of a protein. J. Mol. Biol. 157:105-132.

13.Marck, C. 1988. DNA Strider: a C program for the fast analysis of DNA and protein sequences on the Apple Macintosh family of computers. Nucleic Acids Res. 16:1829-1836.

14.Pace, C.N., F. Vajdos, L. Fee, G. Grimsley and T. Gray. 1995. How to measure and predict the molar absorption coefficient of a protein. Protein Sci. 4:2411-2423.

15.Pearson, W.R. 1990. Rapid and sensitive sequence comparison with FASTP and FASTA. Methods Enzymol. 183:63-98.

16.Read, R.L., D. Davison, J.E. Chappelear and J.S. Garavelli. 1992. GBPARSE: a parser for the GenBank flat-file format with the new feature table format. Comput. Appl. Biosci. 8:407-408.

17.Roberts, R.J. and D. Macelis. 1999. REBASE-restriction enzymes and methylases. Nucleic Acids Res. 27:312-313.

18.Stroustrup, B. 1997. The C++ Programming Language. Third Edition. Addison-Wesley Publishing Company, Reading, MA.

19.Thompson, J.D., D.G. Higgins and T.J. Gibson. 1994. CLUSTAL W: improving the sensitivity of progressive multiple sequence alignment through sequence weighting, position-specific gap penalties and weight matrix choice. Nucleic Acids Res. 22:4673-4680.

20.Walsh, S. and B. Barrell. 1996. The Saccharomyces cerevisiae genome on the World Wide Web. Trends Genet. 12:276-277.

21.Welling, G.W., W.J. Weijer, R. van der Zee and S. Welling-Wester. 1985. Prediction of sequential antigenic regions in proteins. FEBS Lett. 188:215-218.

22.Zhang, J. and T.L. Madden. 1997. PowerBLAST: a new network BLAST application for interactive or automated sequence analysis and annotation. Genome Res. 7:649-656.

Received 26 January 2000; accepted 6 April 2000.

Address correspondence to:

Dr. Steven Graves

P.O. Box 1024

Los Alamos, NM 87544, USA

Internet: steveg@dnassist.com 